\title{
Stationary state and relaxation of artificial irregularities excited in ionospheric heating experiments
}

\author{
N. B ORISOV,,$^{1,2}$ A. SENIOR ${ }^{1}$ and F. HONAR Y \\ ${ }^{1}$ Department of Communication Systems, Lancaster University, Lancaster LA1 4YR, UK \\ (a.senior@lancaster.ac.uk) \\ ${ }^{2}$ Institute of Terrestrial Magnetism, Ionosphere and Radio Waves Propagation, \\ (IZMIRAN) 142190, Troitsk, Moscow Region, Russia
}

(Received 25 September 2003, revised 30 January 2004 and accepted 14 March 2004)

\begin{abstract}
We analytically and numerically discuss the plasma distribution and electron temperature enhancement in striations for a given heating source. It is shown that, in stationary conditions, the reduced plasma concentration and the temperature enhancement should be of the same order of magnitude. We deduce that the electron temperature inside striations cannot greatly exceed that in the heated volume. The elongation of striations is calculated for different transverse scales and heating powers. We show that medium sized irregularities are associated with striations and develop after the formation of striations. The peculiarities of the relaxation process for small-scale and medium sized artificial irregularities are investigated and it is found that the relaxation of small-scale irregularities exhibits two time scales, in qualitative agreement with experimental observations.
\end{abstract}

\section{Introduction}

Small-scale artificial field-aligned irregularities (AFAI) also known as 'striations' appear in the F-region of the ionosphere illuminated by a powerful high-frequency (HF) radio wave. According to theory, striations are formed due to the conversion of the electromagnetic (EM) pump wave into upper-hybrid resonance (UHR) oscillations on small-scale plasma inhomogeneities (Gurevich 1978). The variation of the reduced plasma concentration in striations measured directly on a rocket is of the order of a few per cent (Kelley et al. 1995). Typical transverse scales of striations obtained in the same experiment are about $L_{\perp} \sim 3-10 \mathrm{~m}$, although the total spectrum of excited artificial plasma inhomogeneities is very broad and complicated. Its behaviour for scales from metres down to several centimetres was investigated long ago by backscattering (Minkoff et al. 1974). However, artificial irregularities with larger transversal scales are also excited in heating experiments. In addition to a pronounced maximum for scales $\sim 5 \mathrm{~m}$, a local spectral maximum also exists at scales of the order of a few hundreds of metres (Erukhimov et al. 1987). As for the elongation of AFAI, only rather scant information is available. Different authors give various estimates for the elongation of striations: $L_{\|} \sim 3$ $5 \mathrm{~km}$ (Rao and Thome 1974; Korovin et al. 1983), $L_{\|} \sim 10-15 \mathrm{~km}$ (Jones et al. 1984; 
Kelley et al. 1995). In the existing theory, only crude estimates are obtained based on ambipolar ion diffusion and recombination lifetime (Gurevich et al. 1995a).

For medium-sized irregularities $\left(L_{\perp} \sim 50-200 \mathrm{~m}\right)$, it was found by experiments that they can extend below the reflection height by about $100 \mathrm{~km}$, thus covering the whole F-region (Hedberg et al. 1983; Bakhmet'eva et al. 1989). It is interesting to mention that according to Bakhmet'eva et al. (1989), such irregularities first appear at the heights where striations are formed and later on begin to occupy a larger height interval.

The modern theory of the stationary state of striations predicts a large (3-4 times) increase of the temperature of electrons inside striations and at the same time a very weak variation of plasma concentration (Gurevich et al. 1995a,b). Significant temperature enhancement is indeed measured by incoherent scattering during heating (Robinson et al. 1996; Leyser et al. 2000). Unfortunately, in such experiments it is impossible to distinguish whether the rise of temperature occurs only inside striations or in the bulk plasma. At the same time, Peria et al. (1999) reported rocket observations of the electron temperature enhancement inside striations to be of the order of $100 \mathrm{~K}$. The absolute temperature was not measured in this experiment, but it seems reasonable to assume that this equates to an enhancement of $10 \%$ or less.

In the present paper we give a detailed analytical analysis of the stationary distributions of plasma density and electron temperature in a single striation. The analytic results are supported by a two-dimensional (2D) numerical analysis which we also use to investigate, in a basic form, the effects of vertical inhomogeneity in the ionosphere. We show that, as the temperature and density perturbations are of the same order due to the balance of pressure, the small density perturbations observed in striations imply that the electron temperature inside striations cannot greatly exceed the background temperature in the heated volume. We demonstrate that the elongation of striations can be expected to be greater than previously thought. We also discuss the formation of medium-sized irregularities and show that they are closely connected to striations. Based on our results, the relaxation of AFAI with different transverse scales is investigated.

\section{Basic equations}

In this section we derive equations describing the stationary conditions of AFAI in the F-region of the high-latitude ionosphere. Let us assume that the Earth's magnetic field is directed along the vertical $z$-axis and in the transversal plane the inhomogeneity exists only along the $x$-axis. The results obtained in this paper are valid also in the case where striations have axial symmetry, that is, they are considered as cylinders stretched along the $z$-axis. We suppose that at the heights where striations are formed, collisions with ions play the main role for electrons, $\nu_{\mathrm{ei}} \gg \nu_{\mathrm{en}}$, where $\nu_{\mathrm{ei}}, \nu_{\mathrm{en}}$ are the electron collision frequencies with ions and neutrals. However electrons in the ionosphere lose their energy mainly in nonelastic collisions with neutrals (Gurevich, 1978). The corresponding losses determined by the frequency $\delta_{\mathrm{en}} \nu_{\mathrm{en}}$ are much larger than the losses in collisions with ions $\delta_{\mathrm{ei}} \nu_{\mathrm{ei}}$ up to heights of approximately $300 \mathrm{~km}$. For larger heights, the losses in collisions with ions give a significant contribution to the total losses if the increase of the bulk electron temperature in the heated volume is small enough. Here $\delta_{\text {en }}$ is a loss rate in non-elastic collisions with neutrals, $\delta_{\mathrm{ei}} \approx 2 \mathrm{~m} / M$ is a loss rate in collisions 
with ions and $m, M$ are the masses of the electron and ion. For ions, collisions with neutrals $\nu_{\text {in }}$ play the main role even in the F-region of the ionosphere due to the small ratio $m / M: M \nu_{\text {in }} \gg m \nu_{\text {ei }}$.

To discuss the stationary distribution of plasma and the heating in striations, for electrons we use the hydrodynamic equations in a completely ionized plasma derived by Braginskii (1965) with slight modifications. In the heat equation we insert an additional term $\delta_{\mathrm{en}} \nu_{\mathrm{en}}$ describing the energy losses in non-elastic collisions and in the continuity equation the finite lifetime of electrons $\tau_{\mathrm{r}}$ is retained. As for ions we distinguish two different cases. It is well-known that hydrodynamics can be used only if the transversal scale of plasma inhomogeneity $L_{\perp}$ is much larger than the Larmor radius $L_{\perp} \gg \rho_{\mathrm{Hi}}=v_{\mathrm{Ti}} / \omega_{\mathrm{Hi}}$, where $v_{\mathrm{Ti}}$ is the thermal speed, $\omega_{\mathrm{Hi}}$ is the cyclotron frequency of ions. In the ionosphere $\rho_{\mathrm{Hi}} \approx 3-5 \mathrm{~m}$, which is of the order of a transversal scale of striations. It means that we have to discuss two cases. If $L_{\perp} \gg \rho_{\mathrm{Hi}}$ ions can be described within hydrodynamics; otherwise a kinetic approach should be used.

Electrons in a completely ionized plasma in stationary conditions are described by the following set of equations:

- the continuity equation

$$
\frac{\partial N v_{\mathrm{e} x}}{\partial x}+\frac{\partial N v_{\mathrm{e} z}}{\partial z}+\frac{N-N_{0}}{\tau_{\mathrm{r}}}=0
$$

- the equation of motion

$$
\begin{aligned}
& -\nabla p_{\mathrm{e}}+e N\left(\nabla \varphi-\frac{\mathbf{v}_{\mathrm{e}}}{c} \times \mathbf{H}\right) \\
& -m N \nu_{\mathrm{ei}}\left(0.51 \mathbf{u}_{\|}+\mathbf{u}_{\perp}\right)-0.71 N \nabla_{\|} T_{\mathrm{e}}-\frac{3}{2} \frac{\nu_{\mathrm{ei}} N}{\omega_{\mathrm{He}}} \mathbf{h} \times \nabla T_{\mathrm{e}}=0
\end{aligned}
$$

and;

- the heat equation

$$
\frac{3}{2} \mathbf{v}_{\mathrm{e}} \nabla T_{\mathrm{e}}+T_{\mathrm{e}} \nabla \mathbf{v}_{\mathrm{e}}+\frac{1}{N} \nabla \mathbf{q}_{\mathrm{e}}+\frac{3}{2} \delta_{\mathrm{en}} \nu_{\mathrm{en}}\left(T_{\mathrm{e}}-T_{\mathrm{e} 0}\right)=Q_{\mathrm{T}} .
$$

Here $N$ is the concentration of plasma, $v_{\mathrm{e} x}, v_{\mathrm{e} z}$ are the transversal and the longitudinal components of the velocity of electrons, $\tau_{\mathrm{r}}$ is the recombination lifetime, $p_{\mathrm{e}}=N T_{\mathrm{e}}$ is the pressure, $T_{\mathrm{e}}$ is the temperature of electrons, $N_{0}, T_{\mathrm{e} 0}$ are the undisturbed plasma concentration and the bulk temperature of electrons in the heated volume, $-e$ is the charge of an electron, $\mathbf{E}=-\nabla \varphi$ is the electric field of polarization, $u_{\|}, u_{\perp}$ are the drift velocities of electrons with respect to ions along and across the magnetic field, $\omega_{\mathrm{He}}$ is the cyclotron frequency of electrons, $\mathbf{h}=\mathbf{H} / H$ is the unit vector along the magnetic field line, $Q_{\mathrm{T}}$ is the source of heating, $\mathbf{q}_{\mathrm{e}}$ is the heat flux

$$
\mathbf{q}_{\mathrm{e}}=-\kappa_{\|}^{(\mathrm{e})} \nabla_{\|} T_{\mathrm{e}}-\kappa_{\perp}^{(\mathrm{e})} \nabla_{\perp} T_{\mathrm{e}}-\frac{5}{2} \frac{N T_{\mathrm{e}}}{m \omega_{\mathrm{He}}} \mathbf{h} \times \nabla T_{\mathrm{e}}-0.71 N T_{\mathrm{e}} \mathbf{u}_{\|}+\frac{3}{2} \frac{N T_{\mathrm{e}} \nu_{\mathrm{ei}}}{\omega_{\mathrm{He}}} \mathbf{h} \times \mathbf{u}_{\perp}
$$

where

$$
\kappa_{\perp}^{(\mathrm{e})}=4.66 \frac{N T_{\mathrm{e}} \nu_{\mathrm{ei}}}{m \omega_{\mathrm{He}}^{2}}, \quad \kappa_{\|}^{(\mathrm{e})}=3.16 \frac{N T_{\mathrm{e}}}{m \nu_{\mathrm{ei}}} .
$$

The motion of ions is described by the equation

$$
-\nabla p_{\mathrm{i}}+e N\left(-\nabla \varphi+\frac{\mathbf{v}_{\mathrm{i}}}{c} \times \mathbf{H}\right)-M N \nu_{\text {in }}\left(\mathbf{v}_{\mathrm{i}}-\mathbf{v}_{\mathrm{n}}\right)=0
$$


where $p_{\mathrm{i}}=N T_{\mathrm{i}}$ is the pressure of ions, and $v_{\mathrm{i}}, v_{\mathrm{n}}$ are the speeds of ions and neutral particles. The expression for the longitudinal velocity follows from (2.5)

$$
v_{\mathrm{i} z}=v_{\mathrm{n} z}-\frac{1}{M N \nu_{\mathrm{in}}}\left(\frac{\partial p_{\mathrm{i}}}{\partial z}+e N \frac{\partial \varphi}{\partial z}\right)
$$

and for the transverse $x$-component

$$
v_{\mathrm{i} x}=v_{\mathrm{n} x}+\frac{\nu_{\mathrm{in}}}{\omega_{\mathrm{Hi}}^{2}+\nu_{\mathrm{in}}^{2}}\left(\frac{1}{M N} \frac{\partial p_{\mathrm{i}}}{\partial x}+\frac{e}{M} \frac{\partial \varphi}{\partial x}\right) .
$$

Further, for simplicity we neglect the speed of the neutral wind $\mathbf{v}_{\mathrm{n}}=0$. In this case the continuity equation for ions reduces to

$$
\frac{\partial}{\partial x} D_{\perp}^{(\mathrm{i})} \frac{\partial N}{\partial x}+\frac{\partial}{\partial x} \frac{\sigma_{\perp}^{(\mathrm{i})}}{e} \frac{\partial \varphi}{\partial x}+\frac{\partial}{\partial z} D_{\|}^{(\mathrm{i})} \frac{\partial N}{\partial z}+\frac{\partial}{\partial z} \frac{\sigma_{\|}^{(\mathrm{i})}}{e} \frac{\partial \varphi}{\partial z}+\frac{N-N_{0}}{\tau_{\mathrm{r}}}=0
$$

where

$$
D_{\perp}^{(\mathrm{i})}=\frac{T_{\mathrm{i}}}{M} \frac{\nu_{\mathrm{in}}}{\omega_{\mathrm{Hi}}^{2}+\nu_{\mathrm{in}}^{2}}, \quad D_{\|}^{(\mathrm{i})}=\frac{T_{\mathrm{i}}}{M \nu_{\mathrm{in}}}, \quad \sigma_{\perp}^{(\mathrm{i})}=\frac{e^{2} N}{M} \frac{\nu_{\mathrm{in}}}{\omega_{\mathrm{Hi}}^{2}+\nu_{\mathrm{in}}^{2}}, \quad \sigma_{\|}^{(\mathrm{i})}=\frac{e^{2} N}{M \nu_{\mathrm{in}}} .
$$

According to Gurevich (1978) the lifetime of charged particles $\tau_{\mathrm{r}}$ in the F-region of the ionosphere is of the order of $5 \times 10^{2}-10^{3} \mathrm{~s}$ at night and $10^{2}-5 \times 10^{2} \mathrm{~s}$ during the day. This time is much larger than the period required for the formation of the stationary state of striations (Frolov et al. 1997). Hence we may set $\tau_{\mathrm{r}} \rightarrow \infty$. For typical scales of striations $L_{\|}^{(N)} \geqslant 10^{6} \mathrm{~cm}, L_{\perp}^{(N)} \sim(5-10) \times 10^{2} \mathrm{~cm}$ and the values of the frequencies $\nu_{\text {in }} \sim 2-5 \mathrm{~s}^{-1}, \omega_{\mathrm{Hi}} \sim 10^{2} \mathrm{~s}^{-1}$, we find that the longitudinal terms in (2.8) are much smaller than the transversal terms. As a result, an approximate relation between plasma perturbation and electric potential applies:

$$
\frac{\partial N}{\partial x}+\frac{e N}{T_{\mathrm{i}}} \frac{\partial \varphi}{\partial x}=0
$$

In the case of small-scale plasma inhomogeneities $L_{\perp} \ll \rho_{\mathrm{Hi}}$, it follows from a kinetic approach that ions have, in the first approximation with respect to $L_{\perp} / \rho_{\mathrm{Hi}}$, a Boltzmann distribution (Kadomtsev and Pogutse 1970)

$$
N=N_{0} \exp \left(-\frac{e \varphi}{T_{\mathrm{i}}}\right) .
$$

Hence, in both cases the same relation (2.10) between plasma perturbation and electric potential applies.

Now we concentrate on the equations for electrons. Using (2.10) we eliminate the electric potential $\varphi$ from the system $(2.1)-(2.3)$. It is convenient to introduce dimensionless functions

$$
\frac{N}{N_{0}}=n(z, x), \quad \frac{T_{\mathrm{e}}}{T_{\mathrm{e} 0}}=\tau(z, x) .
$$

Note that this temperature $T_{\mathrm{e} 0}$ can significantly exceed the undisturbed (without heating) temperature of electrons in the ionosphere. In deriving hydrodynamic equations we take into account that collision frequencies $\nu_{\mathrm{ei}}, \nu_{\mathrm{en}}$ depend on the electron temperature

$$
\nu_{\mathrm{ei}}=\nu_{\mathrm{ei}}^{(0)} \tau^{-3 / 2}, \quad \nu_{\mathrm{en}}=\nu_{\mathrm{en}}^{(0)} \tau^{1 / 2}
$$


where $\nu_{\mathrm{ei}}^{(0)}, \nu_{\mathrm{en}}^{(0)}$ are the background collision frequencies in the heated volume (Schunk and Nagy 1978). If the pump frequency is not very far from the critical frequency of the ionosphere, the height variation of the background plasma concentration $N_{0}(z)$ can be neglected. In this case the strongest dependence on the $z$-coordinate exists in the diffusion coefficient $D_{\|}^{(\mathrm{e})}(z)$. Here $D_{\|}^{(\mathrm{e})}=T_{\mathrm{e}} / m \nu_{\mathrm{ei}}$ and $D_{\perp}^{(\mathrm{e})}=T_{\mathrm{e}} \nu_{\mathrm{ei}} / m \omega_{\mathrm{He}}^{2}$ are the longitudinal and transverse diffusion coefficients for electrons. Such a dependence can be modelled in numerical calculations by a linear function $D_{\|}^{(\mathrm{e})}(z)=D_{\|}^{(\mathrm{e})(0)}\left(1+z / L_{z}\right)$. The height dependence of other parameters $\left(D_{\perp}^{(\mathrm{e})}, T_{\mathrm{e} 0}, N_{0}\right)$ is weaker and is therefore neglected. As a result (2.1), (2.3) are presented in the final form

$$
\begin{gathered}
\frac{\partial}{\partial x} \frac{D_{\perp}^{(\mathrm{e})}}{\tau^{3 / 2}}\left[\frac{\partial}{\partial x} n\left(\tau+\frac{T_{\mathrm{i}}}{T_{\mathrm{e} 0}}\right)+\frac{3}{2} n \frac{\partial \tau}{\partial x}\right] \\
+2 \frac{\partial}{\partial z} D_{\|}^{(\mathrm{e})} \tau^{3 / 2}\left[\frac{\partial}{\partial z} n\left(\tau+\frac{T_{\mathrm{i}}}{T_{\mathrm{e} 0}}\right)+0.71 n \frac{\partial \tau}{\partial z}\right]=0 \\
\frac{3.1}{n} \frac{\partial}{\partial x} D_{\perp}^{(\mathrm{e})}\left(n \tau^{-1 / 2} \frac{\partial \tau}{\partial x}\right)+\frac{2.1}{n} \frac{\partial}{\partial z} D_{\|}^{(\mathrm{e})}\left(n \tau^{5 / 2} \frac{\partial \tau}{\partial z}\right) \\
+\frac{1}{n} \frac{\partial}{\partial x} D_{\perp}^{(\mathrm{e})}\left[\tau^{-1 / 2} \frac{\partial}{\partial x} n\left(\tau+\frac{T_{\mathrm{i}}}{T_{\mathrm{e} 0}}\right)\right] \\
+\frac{0.9}{n} \frac{\partial}{\partial z} D_{\|}^{(\mathrm{e})}\left[\tau^{5 / 2} \frac{\partial}{\partial z} n\left(\tau+\frac{T_{\mathrm{i}}}{T_{\mathrm{e} 0}}\right)+0.71 n \tau^{5 / 2} \frac{\partial \tau}{\partial z}\right] \\
+D_{\perp}^{(\mathrm{e})} \tau^{-3 / 2}\left(\frac{\partial \tau}{\partial x}-\frac{2}{3} \frac{\tau}{n} \frac{\partial n}{\partial x}\right)\left[\frac{1}{n} \frac{\partial}{\partial x} n\left(\tau+\frac{T_{\mathrm{i}}}{T_{\mathrm{e} 0}}\right)+\frac{3}{2} \frac{\partial \tau}{\partial x}\right] \\
+2 D_{\|}^{(\mathrm{e})} \tau^{3 / 2}\left(\frac{\partial \tau}{\partial z}-\frac{2}{3} \frac{\tau}{n} \frac{\partial n}{\partial z}\right)\left[\frac{1}{n} \frac{\partial}{\partial z} n\left(\tau+\frac{T_{\mathrm{i}}}{T_{\mathrm{e} 0}}\right)+0.71 \frac{\partial \tau}{\partial z}\right] \\
-\delta_{\mathrm{en}} \nu_{\mathrm{en}} \tau^{1 / 2}(\tau-1)=-\frac{2}{3} \frac{Q_{\mathrm{T}}}{T_{\mathrm{e} 0}} \\
+
\end{gathered}
$$

Equations (2.12)-(2.13) are rather complicated and in the general case can only be solved numerically. If the variations of plasma $\Delta n=n-1$ and temperature enhancement $\Delta \tau=\tau-1$ are small enough, (2.12) and (2.13) are simplified significantly and reduce to the system of linear partial differential equations:

$$
\begin{gathered}
D_{\perp}^{(\mathrm{e})}\left[\left(1+\frac{T_{\mathrm{i}}}{T_{\mathrm{e} 0}}\right) \frac{\partial^{2} n}{\partial x^{2}}+\frac{5}{2} \frac{\partial^{2} \tau}{\partial x^{2}}\right]+2 \frac{\partial}{\partial z} D_{\|}^{(\mathrm{e})}\left[\left(1+\frac{T_{\mathrm{i}}}{T_{\mathrm{e} 0}}\right) \frac{\partial n}{\partial z}+1.71 \frac{\partial \tau}{\partial z}\right]=0 \\
3.1 D_{\perp}^{(\mathrm{e})} \frac{\partial^{2} \tau}{\partial x^{2}}+2.1 \frac{\partial}{\partial z} D_{\|}^{(\mathrm{e})} \frac{\partial \tau}{\partial z}+D_{\perp}^{(\mathrm{e})} \frac{\partial^{2}}{\partial x^{2}}\left[n\left(1+\frac{T_{\mathrm{i}}}{T_{\mathrm{e} 0}}\right)+\tau\right] \\
+0.9 \frac{\partial}{\partial z} D_{\|}^{(\mathrm{e})}\left[\left(1+\frac{T_{\mathrm{i}}}{T_{\mathrm{e} 0}}\right) \frac{\partial}{\partial z} n+1.71 \frac{\partial \tau}{\partial z}\right]-\delta_{\mathrm{en}} \nu_{\mathrm{en}}(\tau-1)=-\frac{2}{3} \frac{Q_{\mathrm{T}}}{T_{\mathrm{e} 0}}
\end{gathered}
$$




\section{Analytical analysis of plasma distribution and temperature enhancement}

Let us assume that the background plasma is vertically homogeneous and perturbations are weak enough $|\Delta n| \ll 1,|\Delta \tau| \ll 1$. In this case the problem can be discussed analytically. Taking into account that each striation is localized in space, that is $\Delta n \rightarrow 0, \Delta \tau \rightarrow 0$ for $|z| \rightarrow \infty$ or $|x| \rightarrow \infty$, after the integration of (2.14), (2.15) along the magnetic field line, we arrive at the following set of ordinary differential equations

$$
\begin{gathered}
\left(1+\frac{T_{\mathrm{i}}}{T_{\mathrm{e} 0}}\right) D_{\perp}^{(\mathrm{e})} \frac{d^{2}}{d x^{2}} \int \Delta n d z+2.5 D_{\perp}^{(\mathrm{e})} \frac{d^{2}}{d x^{2}} \int \Delta \tau d z=0 \\
\left(1+\frac{T_{\mathrm{i}}}{T_{\mathrm{e} 0}}\right) D_{\perp}^{(\mathrm{e})} \frac{d^{2}}{d x^{2}} \int \Delta n d z+4.1 D_{\perp}^{(\mathrm{e})} \frac{d^{2}}{d x^{2}} \int \Delta \tau d z-\delta_{\mathrm{en}} \nu_{\mathrm{en}} \int \Delta \tau d z=\frac{2}{3} \frac{\int Q_{\mathrm{T}} d z}{T_{\mathrm{e} 0}} .
\end{gathered}
$$

The relation between height-integrated perturbation of plasma in striations and the temperature of electrons follows from (3.1):

$$
\int \Delta n d z=-\frac{2.5}{1+T_{\mathrm{i}} / T_{\mathrm{e} 0}} \int \Delta \tau d z .
$$

It is convenient to introduce the vertical scale of the enhanced temperature $L_{\|}^{(T)}$ and the length of striations $L_{\|}^{(N)}$. According to (3.3) the reduced plasma concentration can be estimated as

$$
\Delta n \sim-\frac{2.5}{1+T_{\mathrm{i}} / T_{\mathrm{e} 0}} \frac{L_{\|}^{(T)}}{L_{\|}^{(N)}} \Delta \tau .
$$

The values $L_{\|}^{(T)}$ and $L_{\|}^{(N)}$ should be of the same order. Indeed, in stationary conditions perturbations of temperature at any point cause variations in plasma concentration due to the balance of pressure. Hence, the variation of the reduced plasma concentration in striations is of the same order of magnitude as the variation of the reduced electron temperature. This means that, at least in the linear approximation, the increase of the reduced temperature $\Delta \tau$ causes a similar decrease of the reduced plasma concentration. The system of equations (3.1) and (3.2) reduces to one equation:

$$
1.6 D_{\perp}^{(\mathrm{e})} \frac{d^{2}}{d x^{2}} \int \Delta \tau d z-\delta_{\mathrm{en}} \nu_{\mathrm{en}} \int \Delta \tau d z=-0.7 \frac{\int Q_{\mathrm{T}} d z}{T_{\mathrm{e} 0}+T_{\mathrm{i}}} .
$$

The characteristic scale that enters (3.4) is

$$
L_{\perp}^{*}=\left(\frac{D_{\perp}^{(\mathrm{e})}}{\delta_{\mathrm{en}} \nu_{\mathrm{en}}}\right)^{1 / 2}
$$

If the transverse scale of the heating source in the right-hand side of (3.4) is less than $L_{\perp}^{*}$, the plasma inhomogeneity in stationary conditions still has a scale of the order of $\sim L_{\perp}^{*}$. In the opposite case, the transverse scale of the inhomogeneity is determined by the source

$$
\int \Delta n d z \approx-2.5 \frac{\int Q_{\mathrm{T}} d z}{\left(1+T_{\mathrm{i}} / T_{\mathrm{e} 0}\right) \delta_{\mathrm{en}} \nu_{\mathrm{en}}} .
$$


Integrating (2.14), (2.15) across the magnetic field line, we find an equation for the longitudinal distribution of inhomogeneity

$$
D_{\|}^{(\mathrm{e})} \frac{d^{2}}{d z^{2}} \int \Delta \tau d x-0.3 \delta_{\mathrm{en}} \nu_{\mathrm{en}} \int \Delta \tau d x=-0.3 \frac{\int Q_{\mathrm{T}} d x}{T_{\mathrm{e} 0}} .
$$

This equation contains another characteristic scale that is connected to the length of striations

$$
L_{\|}^{*}=\left(\frac{D_{\|}^{(\mathrm{e})}}{\delta_{\mathrm{en}} \nu_{\mathrm{en}}}\right)^{1 / 2} .
$$

Applying a Fourier transform across the magnetic field line of the form

$$
\Delta n_{\kappa}(z)=\frac{1}{2 \pi L_{\perp}^{*}} \int \Delta n(x, z) \exp i\left(\kappa_{x} \frac{x}{L_{\perp}^{(*)}}\right) d x,
$$

we arrive at the following equation for the Fourier components of plasma perturbation

$$
\begin{gathered}
{\left[L_{\|}^{(*) 4} \frac{d^{4}}{d z^{4}}-\left(1.6 L_{\|}^{(*) 2} \frac{d^{2}}{d z^{2}}+0.4\right)+0.4 \kappa_{x}^{4}+0.2 \kappa_{x}^{2}\right] \Delta n_{\kappa}} \\
=\left(0.4 \kappa_{x}^{2}-0.6 L_{\|}^{(*) 2} \frac{d^{2}}{d z^{2}}\right) \frac{Q_{\kappa}}{\left(\delta_{\mathrm{en}} \nu_{\mathrm{en}}\right)^{2}\left(T_{\mathrm{e} 0}+T_{\mathrm{i}}\right)} .
\end{gathered}
$$

Here $\kappa_{x}=k_{x} L_{\perp}^{*}$ is the dimensionless wave number along the $x$-axis. If the heating source has a large transverse scale $\left(\kappa_{x}^{2} \ll 1\right)$ it follows from $(3.9)$ that the length of a striation is determined by

$$
\left(-L_{\|}^{(*) 2} \frac{d^{2}}{d z^{2}}+0.4\right) \Delta n_{\kappa}=0.6 \frac{Q_{\kappa}}{\left(\delta_{\mathrm{en}} \nu_{\mathrm{en}}\right)^{2}\left(T_{\mathrm{e} 0}+T_{\mathrm{i}}\right)} .
$$

It is seen from (3.10) that the maximal length of a striation in the vertically homogeneous ionosphere is approximately equal to $3 L_{\|}^{*}$. It is achieved for striations with not too small transverse scales $L_{\perp}^{(N)}>L_{\perp}^{(*)}$. With the decrease of the transverse scale the length of a striation changes. It is seen that the solution of (3.9) decreasing for $|z| \rightarrow \infty$ takes the form

$$
\Delta n_{\kappa}(z)=C_{1}\left(\kappa_{x}\right) \exp \left(-p_{1} \frac{|z|}{L_{\|}^{(*)}}\right)+C_{2}\left(\kappa_{x}\right) \exp \left(-p_{2} \frac{|z|}{L_{\|}^{(*)}}\right),
$$

where $p_{1,2}$ are the following

$$
p_{1,2}^{2}=0.8 \kappa_{x}^{2}+0.2 \pm \sqrt{0.24 \kappa_{x}^{4}+0.16 \kappa_{x}^{2}+0.04},
$$

and $C_{1}\left(\kappa_{x}\right), C_{2}\left(\kappa_{x}\right)$ are determined by the source in the right-hand side of (3.9). It is interesting to mention that the solution (3.11) determines striations with two different lengths (due to the fact that $p_{1}>p_{2}$ ). The first solution (with the coefficient $p_{1}$ in the exponent) describes a striation that is definitely shorter than a striation with large transverse scale (determined by (3.10)). The second solution corresponds to a striation with a length that is close enough to the length of a broad striation. This means that in the general case the length of striations increases with the growth of the transversal scale. This result is in qualitative agreement with the experimental data (Frolov et al. 1997). 
It is possible to find an analytical solution of the nonlinear system (2.12), (2.13) in some approximation. We suppose once more that the background plasma is vertically homogeneous and only perturbations with large transverse scales $L_{\perp}^{(N)} \gg L_{\perp}^{*}$ are to be considered. In this case a relation between the reduced plasma perturbation $n$ and the electron temperature $\tau$ follows from (2.12)

$$
n=\frac{\left(1+T_{\mathrm{i}} / T_{\mathrm{e} 0}\right)^{\alpha}}{\left(\tau+T_{\mathrm{i}} / T_{\mathrm{e} 0}\right)^{\alpha}}, \quad \alpha=1.71 .
$$

Usually in the F-region of the ionosphere the temperature of ions is smaller than the temperature of electrons and so the ratio $T_{\mathrm{i}} / T_{\mathrm{e} 0} \ll 1$ is neglected. After the substitution of (3.13) into the heat equation (2.13), we arrive at the following equation

$$
\frac{d^{2} f}{d \zeta^{2}}-0.9\left(f^{-1 / 9}-f^{-2 / 3}\right)=-0.6 \frac{\int Q_{\mathrm{T}}(x) d z}{\delta_{\mathrm{en}} \nu_{\mathrm{en}} T_{\mathrm{e} 0}} \delta(z),
$$

where $f=\tau^{1.8}, \zeta=z / L_{\|}^{*}$. As the length of the temperature perturbation is much larger than the range of heights where the heating source is localized, we are able to present the source in the right-hand side of (3.14) as a $\delta$-function. The homogeneous equation (3.14) reduces to a differential equation of the first order

$$
\frac{d f}{d \zeta}=\mp \sqrt{2\left(f^{8 / 9}-1\right)-5.4\left(f^{1 / 3}-1\right)}
$$

where the signs $\mp$ correspond to $\zeta>0$ and $\zeta<0$. From (3.14) and (3.15) we arrive at the following equation for the maximal reduced temperature $\tau_{0}(x)$ reached in a cross section of a striation at $\zeta=0$

$$
\tau_{0}^{1.6}-2.7 \tau_{0}^{0.6}+1.7=0.05 \frac{\left(\int Q_{\mathrm{T}}(z, x) d z\right)^{2}}{\left(\delta_{\mathrm{en}} \nu_{\mathrm{en}} L_{\|}^{*} T_{\mathrm{e} 0}\right)^{2}} .
$$

It is seen from (3.16) that with the growth of the heating source power $Q_{\mathrm{T}}$ the temperature of electrons also increases. For high powers the maximal reduced temperature rises as

$$
\tau_{0} \propto\left(\int Q_{\mathrm{T}} d z\right)^{5 / 4} .
$$

The distribution of the enhanced electron temperature along a striation is obtained numerically from (3.15). The results of computations are presented in Fig. 1 for different values $\tau_{01}=3, \tau_{02}=1$.1. It is seen that the length of the perturbation is very large $L_{\|}^{(N)} \geqslant 100 \mathrm{~km}$. It can be even more for a high maximal temperature enhancement $\tau_{0} \gg 1$. This conclusion is based on the assumption that the usual collision frequency $\nu_{\mathrm{ei}}$ for electrons can be used. As the plasma inside striations is in a turbulent state due to the excitation of UHR oscillations, it is quite obvious that effective collision frequency $\nu_{\mathrm{e}}^{(\mathrm{eff})}$ should be introduced in our calculations instead of $\nu_{\mathrm{ei}}$. It is well known that the effective collision frequency substantially exceeds the usual collision frequency for high levels of turbulence. Therefore, the length of a striation should be smaller than that obtained in Fig. 1.

It is important to mention that the distribution of plasma determined by (3.13) shows that strong enhancement of the electron temperature causes the formation of a very deep plasma cavity. Since experiments show rather weak plasma depletion 

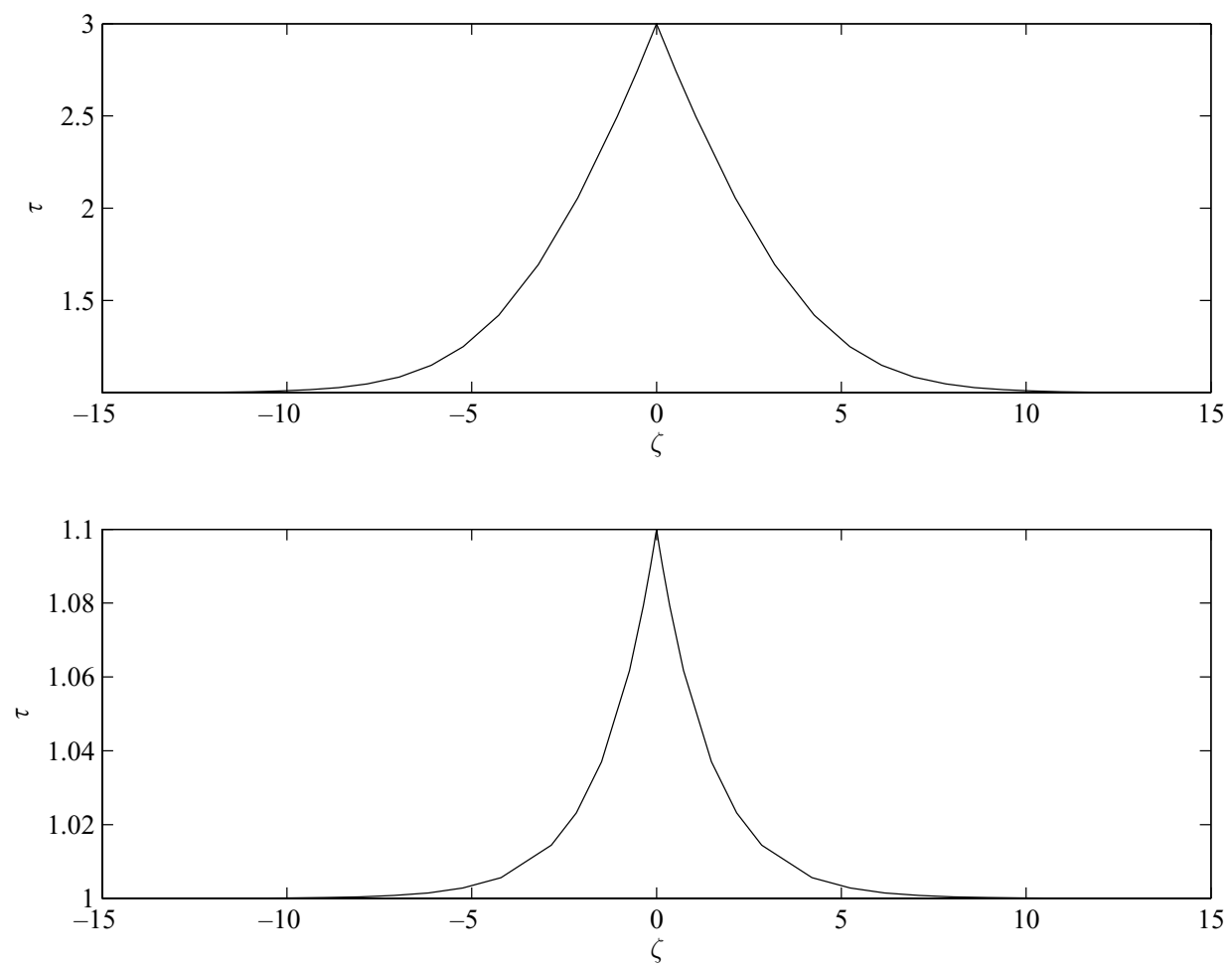

Figure 1. Solutions of (3.15) for $\tau_{01}=3, \tau_{02}=1.1$.

in striations, our results predict that the reduced temperature enhancement in striations is also small. Nevertheless, the increase of the background temperature $T_{\mathrm{e} 0}$ compared to the non-disturbed electron temperature in the ionosphere can be significant.

\section{Numerical analysis of the distribution of plasma and electron temperature in striations}

Numerical analysis allows us to consider the full 2D solution of the nonlinear equations $(2.12),(2.13)$. The purpose of this analysis is to validate the analytical approximations of the previous section and investigate the effect of vertical inhomogeneity of the ionosphere on the solutions in a basic form. We note that computational modelling would, in principle, allow a fully self-consistent model of the development of striations to be constructed. However, many processes must be taken into account, in particular, the plasma turbulence in the region where the EM pump wave couples to the upper-hybrid waves. A comprehensive model would have to simultaneously address small-scale features such as this, and the propagation of the pump wave and the plasma transport processes on the large scale. Before such a model can be constructed it is important to understand at a basic level the processes involved. Here we confine ourselves to discussing in detail the nature of the transport processes associated with striations for a specified heating source. 
Numerical modelling of plasma and electron temperature distribution in the ionosphere heated by powerful radio waves has been undertaken many times, e.g. Hansen et al. (1990), Blaunstein (1996, 1997), Guzdar et al. (1998), Gondarenko et al. (1999). These papers either considered large-scale modification in which transverse transport can be neglected or only included it approximately. In our case, transverse transport is very important because of the small scale sizes of striations across the magnetic field and our model fully includes transverse transport effects.

Equations (2.12) and (2.13) were solved numerically for $n$ and $\tau$ using a finitedifference method on a non-uniform rectangular $(x, z)$ grid. Since $(2.13)$ is nonlinear in $\tau$, an iterative method was used in which a linearized version of (2.13) was solved at each step such that the solution converged to the solution of the nonlinear equation (2.13). The heating source $Q_{\mathrm{T}}$ is modelled by a two-dimensional Gaussian function

$$
Q_{\mathrm{T}}(x, z)=Q_{0} \exp \left(-\left(x / L_{\perp}\right)^{2}-\left(z / L_{\|}\right)^{2}\right),
$$

where $Q_{0}$ represents the peak value of the heating source. In the numerical solutions presented here we take $L_{\|}=1 \mathrm{~km}$. The coupled equations are to be solved subject to the boundary conditions $n, \tau \rightarrow 1$ as $|x|,|z| \rightarrow \infty$. Since we cannot have an infinite grid in our numerical scheme, we approximate these boundary conditions by requiring $n=1, \tau=1$ on the boundary of our solution grid whose limits are made sufficiently large so as to be effectively at infinity for the case under consideration.

To begin with, we assume that the background plasma is completely homogeneous. Except where stated otherwise, we use the following conditions in the numerical solutions presented here: $D_{\|}^{(\mathrm{e})}=6 \times 10^{12} \mathrm{~cm}^{2} \mathrm{~s}^{-1}, D_{\perp}^{(\mathrm{e})}=330 \mathrm{~cm}^{2} \mathrm{~s}^{-1}, T_{\mathrm{i}}=$ $1100 \mathrm{~K}, T_{\mathrm{e} 0}=2500 \mathrm{~K}, \delta=1 \times 10^{-3}, \nu_{\mathrm{en}}=40 \mathrm{~s}^{-1}$. These conditions are based on nighttime experiments at Tromsø, Norway and the high value of $T_{\mathrm{e} 0}$ represents the observed bulk-heating of the plasma.

Figure 2 shows the longitudinal distributions of $n$ and $\tau$ for striations with $L_{\perp}=3 \mathrm{~m}$ with different peak values of the heating source. It is clear that increasing the heating power results in hotter striations with deeper density depletions. We should stress that the large depletions indicated in this figure are not to be expected in practice and only demonstrate what can be expected for a given heat source. As noted above, in reality the heating source in a striation (upper-hybrid waves) is a function of many processes and we do not attempt to self-consistently estimate it here. It can also be seen that the length of the striations increases with increasing heating power. For large temperature enhancements, the decay of temperature and density perturbations along the field line is no longer exponential. The relationship between the peak electron temperature $\tau_{0}$, peak density depletion $n_{0}$ and the heating power is presented in Fig. 3. The temperature enhancement and density depletion can be seen to be of a similar order as expected. Note that the curves show some tendency towards saturation as the heating power is increased.

The effect of changing the transverse scale size of the heating source $L_{\perp}$ can be seen in Fig. 4. The longitudinal scale of the temperature and density profiles $L_{\|}^{(T)}$ and $L_{\|}^{(N)}$ can be seen to increase as the striations become broader. This is to be expected since, for narrower striations, the effect of transverse heat diffusion becomes significant compared to longitudinal diffusion and causes the perturbation 

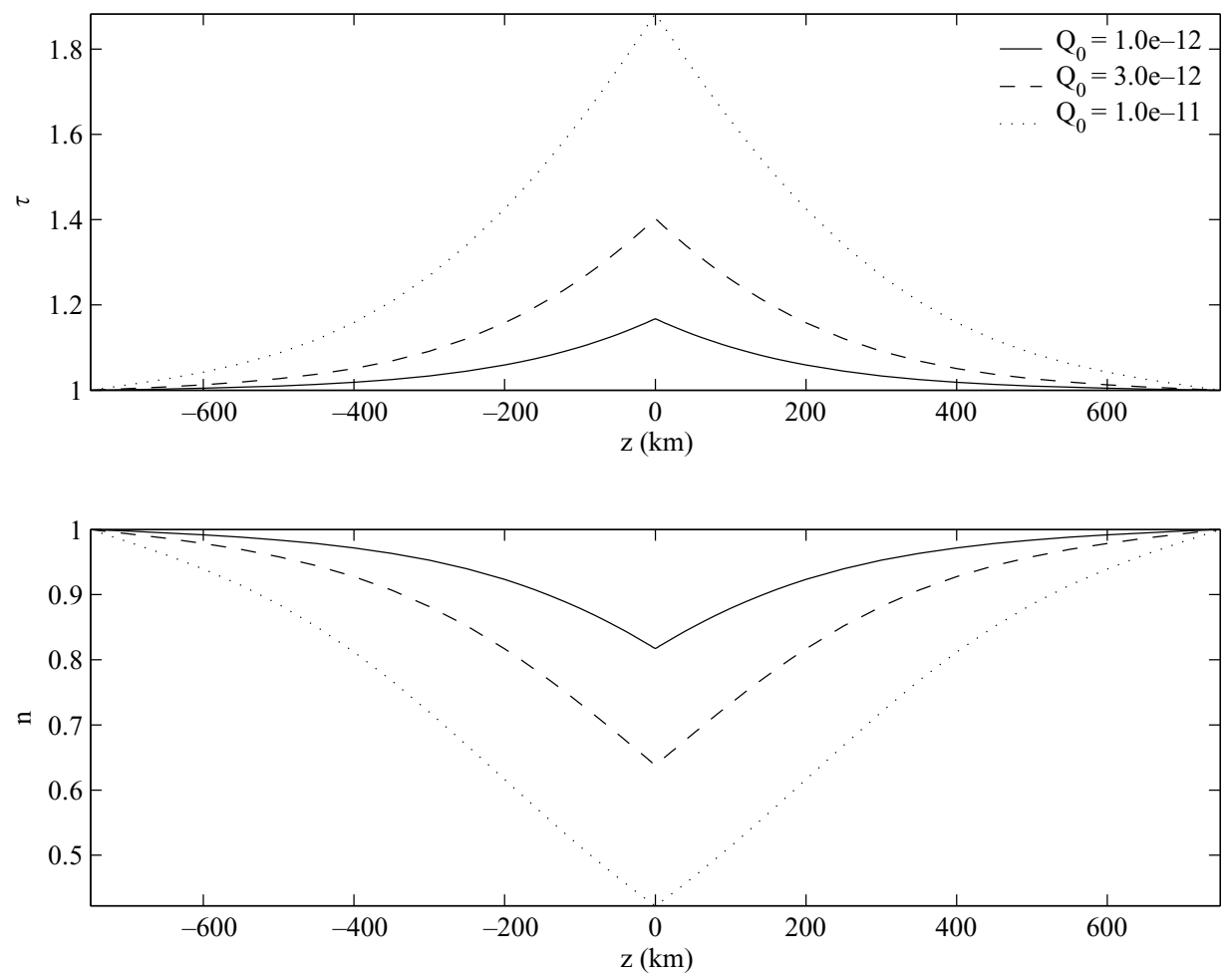

Figure 2. Longitudinal profiles of reduced electron temperature $\tau$ (top panel) and reduced density $n$ (bottom panel) for three different heating powers. Here $L_{\perp}=3 \mathrm{~m}$.

to decay more rapidly along the field line. The longitudinal profiles for transverse scales of 3 and $6 \mathrm{~m}$ almost coincide. This demonstrates that once $L_{\perp}$ exceeds a certain scale, the transverse scale no longer determines the length of striations. The transverse distributions of $n$ and $\tau$ for $L_{\perp}=0.5,1 \mathrm{~m}$ in Fig. 4 are very similar. This corresponds to the effect described in Sec. 3 that for scales $L_{\perp}<L_{\perp}^{*}$, the transverse scale is dominated by diffusion rather than by the scale of the heating source. These results are summarized in Fig. 5 which shows the variation of $L_{\|}^{(T)}$ and $L_{\|}^{(N)}$ as functions of $L_{\perp}$. Here we have defined $L_{\|}^{(T)}$ and $L_{\|}^{(N)}$ as being the $e$-folding distances of the longitudinal profiles of $\Delta \tau$ and $\Delta n$, respectively.

The numerical approach allows us to investigate the effects of vertical inhomogeneity on the parameters of striations. As discussed in Sec. 2, we may model the vertical inhomogeneity by making the longitudinal diffusion coefficient $D_{\|}^{(\mathrm{e})}$ a linear function of $z$ :

$$
D_{\|}^{(\mathrm{e})}=D_{\|}^{(\mathrm{e})(0)}\left(1+z / L_{z}\right)
$$

where $L_{z}$ is the vertical scale length of inhomogeneity and $D_{\|}^{(\mathrm{e})(0)}$ is the diffusion coefficient at $z=0$. Figure 6 shows the results of such calculations for different scales $L_{z}$. It is clear that for small $L_{z}$, striations are greatly elongated in the topside ionosphere and shortened in the bottomside.

In Fig. 7 we have investigated the effect of different ionospheric conditions on the elongation of striations. We have considered two cases intended to represent 


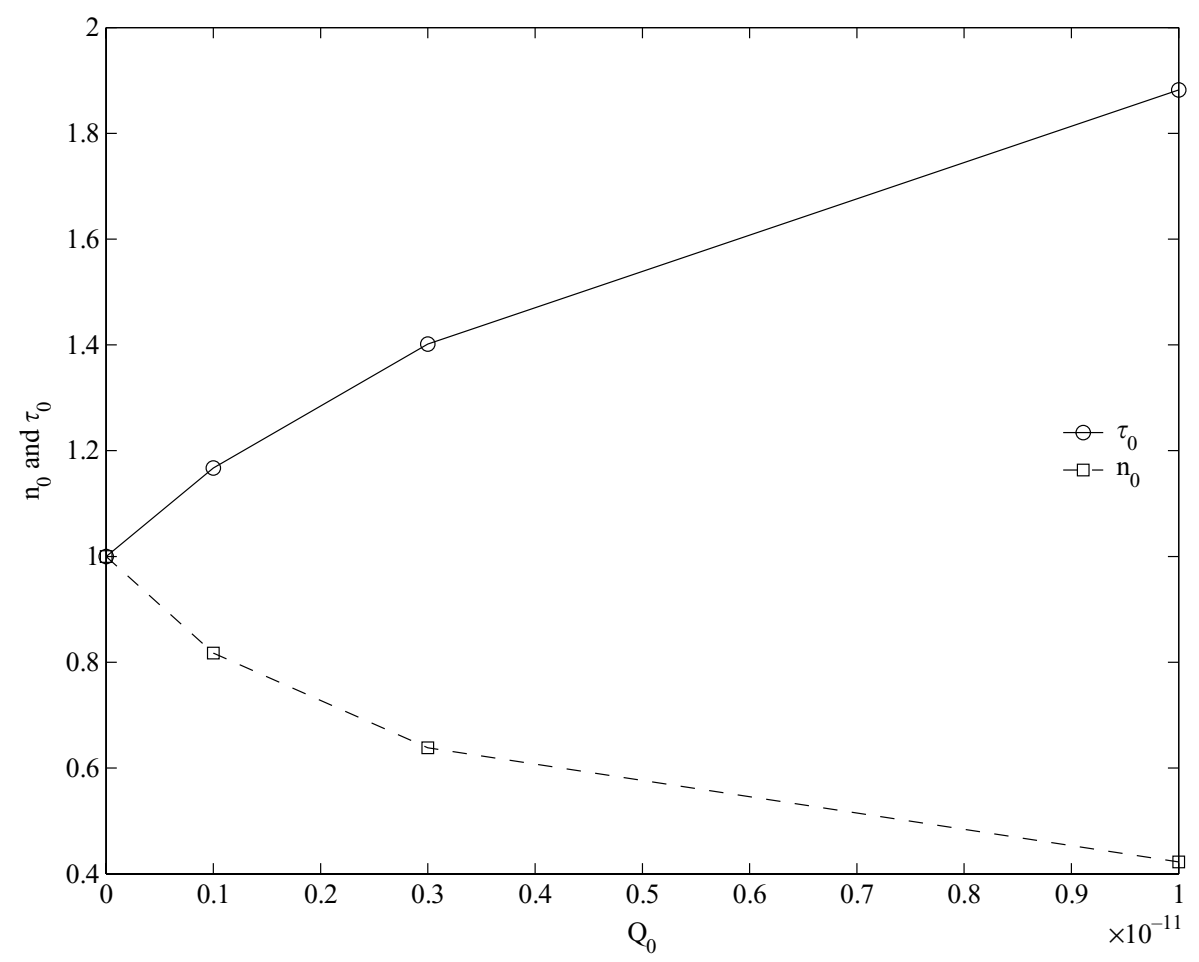

Figure 3. Variation of $\tau_{0}$ and $n_{0}$ with heating power for $L_{\perp}=3 \mathrm{~m}$.

Table 1. Ionospheric conditions used in the solutions of Fig. 7.

\begin{tabular}{cccccc}
\hline Case & $T_{\mathrm{e} 0}(\mathrm{~K})$ & $T_{\mathrm{i}}(\mathrm{K})$ & $D_{\|}^{(\mathrm{e})}\left(\mathrm{cm}^{2} \mathrm{~s}^{-1}\right)$ & $D_{\perp}^{(\mathrm{e})}\left(\mathrm{cm}^{2} \mathrm{~s}^{-1}\right)$ & $\nu_{\mathrm{en}}\left(\mathrm{s}^{-1}\right)$ \\
\hline \multirow{2}{*}{ Day } & 1250 & 1100 & $3.8 \times 10^{11}$ & 1200 & 75 \\
& 2500 & 1100 & $2.1 \times 10^{12}$ & 880 & 100 \\
Night & 1250 & 1100 & $1 \times 10^{12}$ & 460 & 30 \\
& 2500 & 1100 & $6 \times 10^{12}$ & 330 & 40 \\
\hline
\end{tabular}

'typical' daytime and nighttime conditions. For each of these cases we have considered two values for the bulk electron temperature $T_{\mathrm{e} 0}$. These two values represent situations with and without the growth of $T_{\mathrm{e} 0}$ in the whole heated volume. The complete sets of conditions used are shown in Table 1. It is clear that a greater value of $T_{\mathrm{e} 0}$ increases the elongation of striations in both daytime and nighttime conditions. The effect of the factor of two change in $T_{\mathrm{e} 0}$ is quite considerable. It is also evident that striations can be expected to be less elongated in daytime than nighttime conditions. In addition, we note that the density depletion associated with the temperature enhancement is of the same order in all cases and is of the same order as the temperature enhancement itself.

In summary, the numerical results confirm the findings of the simplified analytical approach. In particular, we find that the length of striations increases with increasing transverse scale as predicted from the linearized analytical solution. The numerical approach has also allowed us to investigate the effects of vertical 

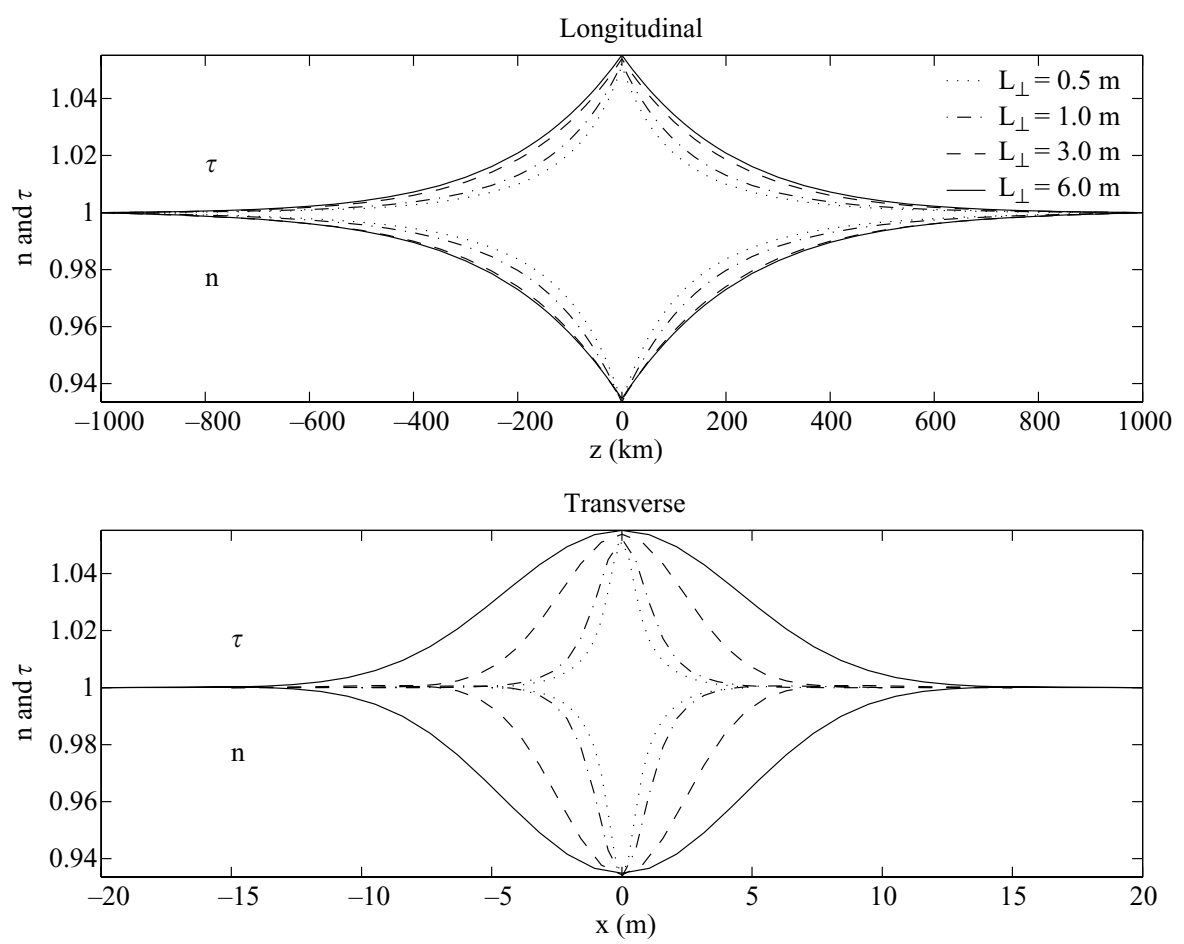

Figure 4. Longitudinal (top panel) and transverse (bottom panel) profiles of $n$ and $\tau$ for different transverse scale sizes of the heating source $L_{\perp}$. The value of $Q_{0}$ has been adjusted to achieve the same peak temperature $\tau_{0}$ in each case.

inhomogeneity which is necessary in order to compare the theoretical predictions with observations made in a real ionosphere.

\section{Formation of medium-sized plasma irregularities}

It is interesting to mention that the equations presented in Sec. 2 also describe plasma inhomogeneities with quite different transverse scales. To demonstrate it in the most simple form we assume once more that the plasma is vertically homogeneous and the perturbations $\Delta \tau, \Delta n$ are weak enough. We present the total plasma perturbation associated with the heating inside a striation as a sum $\Delta n=\Delta n_{1}+\Delta n_{2}$, where $\Delta n_{1}$ is a small-scale plasma depletion (a striation) and $\Delta n_{2}$ is an additional inhomogeneity. Suppose that this inhomogeneity has a rather large transverse scale $L_{\perp}^{(N)} \gg\left(\nu_{\mathrm{ei}} / \omega_{\mathrm{He}}\right) L_{\|}^{(T)}$. We consider such an inhomogeneity as medium-sized. It will be shown that such inhomogeneities are weaker than striations $\left|\Delta n_{2}\right| \ll\left|\Delta n_{1}\right|$. For small-scale perturbations the results obtained in the previous sections are valid. Let us consider the distribution of the electron temperature inside a striation as a given function of coordinates $\Delta \tau(x, z)$. In this case we have two continuity equations, one each for electrons and ions that contain two unknown functions $\Delta n$ and $\varphi$. In the right-hand side of the continuity equation for electrons, the temperature enhancement $\Delta \tau$ is present as the source of perturbation. If the electric potential is eliminated from the continuity equations, we arrive at 


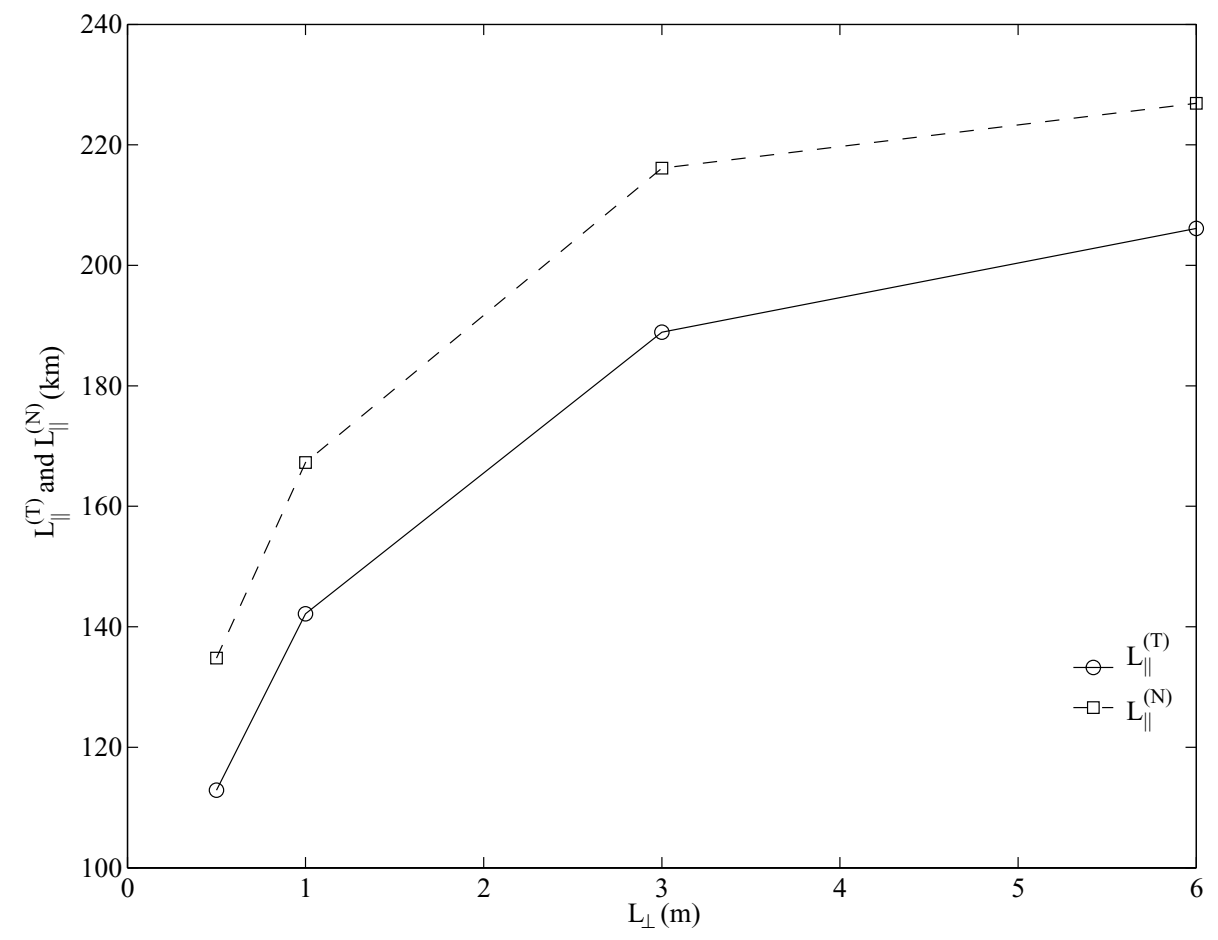

Figure 5. The variation of $L_{\|}^{(T)}$ and $L_{\|}^{(N)}$ as functions of $L_{\perp}$, derived from the profiles of Fig. 4.

an equation of the fourth order

$$
\begin{gathered}
{\left[\left(1+\frac{T_{\mathrm{i}}}{T_{\mathrm{e} 0}}\right) D_{\perp}^{(\mathrm{e})} D_{\perp}^{(\mathrm{i})} \frac{\partial^{4}}{\partial x^{4}}+\left(2 D_{\|}^{(\mathrm{e})} D_{\perp}^{(\mathrm{i})}\left(1+\frac{T_{\mathrm{i}}}{T_{\mathrm{e} 0}}\right) \frac{\partial^{2}}{\partial z^{2}}-\frac{D_{\perp}^{(\mathrm{i})}}{\tau_{\mathrm{r}}}\right) \frac{\partial^{2}}{\partial x^{2}}\right] \Delta n} \\
-\left[2 D_{\|}^{(\mathrm{i})} D_{\|}^{(\mathrm{e})}\left(1+\frac{T_{\mathrm{i}}}{T_{\mathrm{e} 0}}\right) \frac{\partial^{4}}{\partial z^{4}}+\frac{2}{\tau_{\mathrm{r}}} \frac{T_{\mathrm{i}}}{T_{\mathrm{e} 0}} D_{\|}^{(\mathrm{e})} \frac{\partial^{2}}{\partial z^{2}}\right] \Delta n \\
=-\left(2.5 D_{\perp}^{(\mathrm{e})} \frac{\partial^{2}}{\partial x^{2}}+3.4 D_{\|}^{(\mathrm{e})} \frac{\partial^{2}}{\partial z^{2}}\right)\left(D_{\perp}^{(\mathrm{i})} \frac{\partial^{2}}{\partial x^{2}}+D_{\|}^{(\mathrm{i})} \frac{\partial^{2}}{\partial z^{2}}\right) \Delta \tau
\end{gathered}
$$

This equation describes plasma inhomogeneities with two different transverse scales. Here we are only interested in medium-sized inhomogeneities, for which an approximate equation follows from (5.1):

$$
\left(D_{\perp}^{(\mathrm{i})} \frac{\partial^{2}}{\partial x^{2}}+D_{\|}^{(\mathrm{i})} \frac{\partial^{2}}{\partial z^{2}}-\frac{T_{\mathrm{i}}}{T_{\mathrm{i}}+T_{\mathrm{e} 0}} \frac{1}{\tau_{\mathrm{r}}}\right) \Delta n_{2}=-3.4\left(D_{\perp}^{(\mathrm{i})} \frac{\partial^{2}}{\partial x^{2}}+D_{\|}^{(\mathrm{i})} \frac{\partial^{2}}{\partial x^{2}}\right) \Delta \tau \text {. }
$$

Note that we have retained the lifetime $\tau_{\mathrm{r}}$ in (5.2) because medium-sized irregularities are not formed as quickly as striations. Taking into account that the length of the perturbation determined by the longitudinal diffusion of electrons $L_{\|}^{(N)} \sim\left(D_{\|}^{(\mathrm{e})} / \delta_{\mathrm{en}} \nu_{\mathrm{en}}\right)^{1 / 2}$ is very large compared to the scale $\left(D_{\|}^{(\mathrm{i})} \tau_{\mathrm{r}}\right)^{1 / 2}$, it is possible to neglect the longitudinal diffusion in the left-hand side of (5.2). However, we are not able to neglect the similar term in the right-hand side of (5.2) because the term 

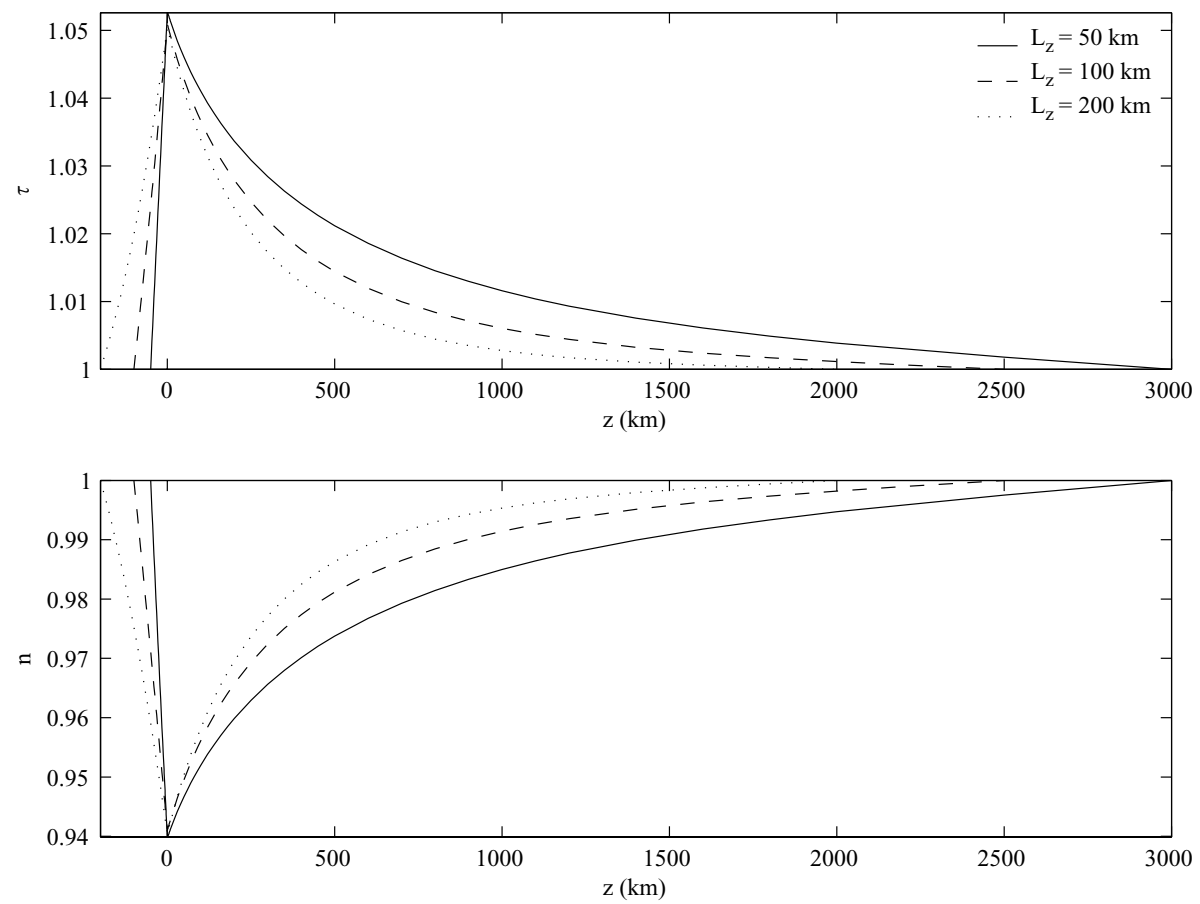

Figure 6. Longitudinal profiles of $\tau$ (top panel) and $n$ (bottom panel) for different scales of vertical inhomogeneity $L_{z}$. The value of $Q_{0}$ has been adjusted to achieve the same peak temperature $\tau_{0}$ in each case. For clarity, the full domain of the solutions is not shown.

$D_{\perp}^{(\mathrm{i})}\left(\partial^{2} / \partial x^{2}\right) \Delta \tau$ gives no contribution to the formation of a medium-sized plasma inhomogeneity. As a result we arrive at the equation

$$
\left(D_{\perp}^{(\mathrm{i})} \frac{\partial^{2}}{\partial x^{2}}-\frac{T_{\mathrm{i}}}{T_{\mathrm{i}}+T_{\mathrm{e} 0}} \frac{1}{\tau_{\mathrm{r}}}\right) \Delta n_{2}=-3.4 D_{\|}^{(\mathrm{i})} \frac{\partial^{2}}{\partial z^{2}} \Delta \tau .
$$

It follows from (5.3) that each striation is surrounded by a medium-sized plasma depletion. The depletion described by (5.3) is weak. In stationary conditions

$$
\Delta n \sim-3.4 \frac{D_{\|}^{(\mathrm{i})}}{D_{\|}^{(\mathrm{e})}} \frac{\tau_{\mathrm{r}}}{\tau_{\mathrm{T}}} \Delta \tau
$$

It is worthwhile to numerically estimate the parameters of a cavity. For typical values $\tau_{\mathrm{T}} \sim 10 \mathrm{~s}, \tau_{\mathrm{r}} \sim 5 \times 10^{2} \mathrm{~s}$, we find $L_{\perp, \mathrm{i}}^{(N)} \sim 100-200 \mathrm{~m}, \Delta n_{2} \sim(0.3-1) \times 10^{-3}$. Each medium-sized plasma depletion acts as a seed cavity for thermal self-focusing of the EM pump wave. It is important that seed plasma inhomogeneities are formed at the heights where striations are developed and not at the bottom of the F-layer. So, self-focusing should start at such heights (in the vicinity of the UHR level). This result is in agreement with the experimental data (Bakhmet'eva et al. 1989). Later on, self-focusing causes significant elongation of medium-sized cavities. It is possible to expect that in stationary conditions medium-sized depletions extend up to $100 \mathrm{~km}$ below the reflection height of the pump wave as measured by Hedberg et al. (1983). 

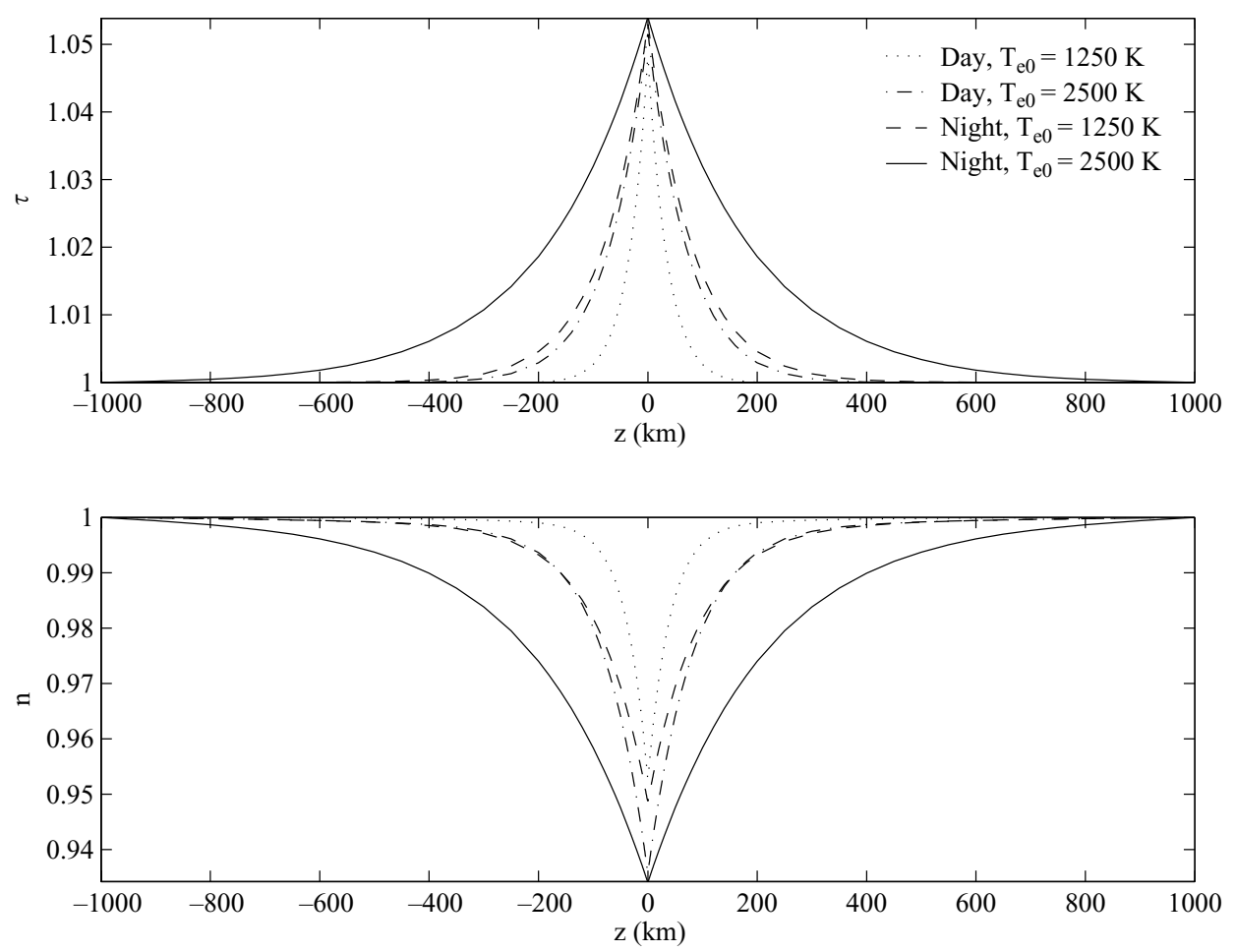

Figure 7. Longitudinal profiles of $\tau$ (top panel) and $n$ (bottom panel) for different ionospheric conditions. The value of $Q_{0}$ has been adjusted to achieve the same peak temperature $\tau_{0}$ in each case.

\section{Relaxation of artificial plasma irregularities}

Our results allow us to investigate the relaxation of AFAI. For this purpose we apply a Fourier transform to the linearized continuity equations for electrons and ions and the heat equation. Note that time derivatives should be retained to investigate the relaxation process. So we start with the following system of equations:

$$
\frac{\partial \Delta n_{k}}{\partial t}+k_{x}^{2} D_{\perp}^{(\mathrm{e})}\left(\Delta n_{k}-\Phi_{k}+2.5 \Delta \tau_{k}\right)+2 k_{z}^{2} D_{\|}^{(\mathrm{e})}\left(\Delta n_{k}-\Phi_{k}+1.7 \Delta \tau_{k}\right)+\frac{\Delta n_{k}}{\tau_{\mathrm{r}}}=0
$$

$$
\begin{gathered}
\frac{\partial \Delta n_{k}}{\partial t}+k_{x}^{2} D_{\perp}^{(\mathrm{i})}\left(\Delta n_{k}+\frac{T_{\mathrm{e} 0}}{T_{\mathrm{i}}} \Phi_{k}\right)+k_{z}^{2} D_{\|}^{(\mathrm{i})}\left(\Delta n_{k}+\frac{T_{\mathrm{e} 0}}{T_{\mathrm{i}}} \Phi_{k}\right)+\frac{\Delta n_{k}}{\tau_{\mathrm{r}}}=0 \\
\frac{\partial \Delta \tau_{k}}{\partial t}+k_{x}^{2} D_{\perp}^{(\mathrm{e})}\left(6.16 \Delta \tau_{k}+1.5 \Delta n_{k}-1.5 \Phi_{k}\right) \\
+k_{z}^{2} D_{\|}^{(\mathrm{e})}\left(7.54 \Delta \tau_{k}+1.4 \Delta n_{k}-1.4 \Phi_{k}\right)+\frac{\Delta \tau_{k}}{\tau_{\mathrm{T}}}=\frac{Q_{\mathrm{T}, k}}{T_{\mathrm{e} 0}}
\end{gathered}
$$


where $\Phi_{k}=\left(e / T_{\mathrm{e} 0}\right) \varphi_{k}, \tau_{\mathrm{T}}=\left(\delta_{\mathrm{en}} \nu_{\mathrm{en}}\right)^{-1}$ and $Q_{\mathrm{T}, k}$ is the Fourier transform of the heating source $Q_{\mathrm{T}}$. An important approximate relation follows from (6.1) and (6.2):

$$
\eta\left(\Delta n_{k}+\frac{T_{\mathrm{e} 0}}{T_{\mathrm{i}}} \Phi_{k}\right)=\Delta n_{k}-\Phi_{k}+1.7 \Delta \tau_{k},
$$

where $\eta=k_{x}^{2} D_{\perp}^{(\mathrm{i})} / 2 k_{z}^{2} D_{\|}^{(\mathrm{e})}$. For small-scale irregularities

$$
L_{\perp}^{(N)} \ll L_{\perp}^{(\mathrm{cr})}=L_{\|}^{(N)}\left(\frac{D_{\perp}^{(\mathrm{i})}}{D_{\|}^{(\mathrm{e})}}\right)^{1 / 2},
$$

the coefficient $\eta$ is large $\eta \gg 1$. Hence, the following relation between plasma perturbation and electric potential applies:

$$
\Delta n_{k}+\frac{T_{\mathrm{e} 0}}{T_{\mathrm{i}}} \Phi_{k}=0
$$

For medium-sized irregularities $L_{\perp}^{(N)} \gg L_{\perp}^{(\mathrm{cr})}$ the coefficient $\eta$ is small. In this case, another relation is valid instead of $(6.5)$ :

$$
\Delta n_{k}-\Phi_{k}+1.7 \Delta \tau_{k}=0
$$

The critical scale $L_{\perp}^{(\mathrm{cr})}$ can be estimated if we take into account that $L_{\|}^{(N)} \approx L_{\|}^{(T)} \sim$ $\left(D_{\|}^{(\mathrm{e})} \tau_{\mathrm{T}}\right)^{1 / 2}$ :

$$
L_{\perp}^{(\mathrm{cr})}=\left(D_{\perp}^{(\mathrm{i})} \tau_{\mathrm{T}}\right)^{1 / 2}
$$

In the F-region of the ionosphere, $L_{\perp}^{(\mathrm{cr})}$ is of the order of $\sim 10 \mathrm{~m}$. This is in qualitative agreement with the experimental data (Frolov et al. 1997).

To investigate the relaxation of a medium-sized depletion, one should substitute the relation $(6.6)$ into $(6.2),(6.3)$. As a result, we arrive at the equations

$$
\begin{gathered}
\frac{\partial \Delta n_{k}}{\partial t}+\left(1+\frac{T_{\mathrm{e} 0}}{T_{\mathrm{i}}}\right)\left(k_{x}^{2} D_{\perp}^{(\mathrm{i})}+k_{z}^{2} D_{\|}^{(\mathrm{i})}\right) \Delta n_{k}=-1.7 \frac{T_{\mathrm{e} 0}}{T_{\mathrm{i}}}\left(k_{x}^{2} D_{\perp}^{(\mathrm{i})}+k_{z}^{2} D_{\|}^{(\mathrm{i})}\right) \Delta \tau_{k} \\
\frac{\partial \Delta \tau_{k}}{\partial t}+\left(5.2 k_{x}^{2} D_{\perp}^{(\mathrm{e})}+k_{z}^{2} D_{\|}^{(\mathrm{e})}\right) \Delta \tau_{k}+\frac{\Delta \tau_{k}}{\tau_{\mathrm{T}}}=\frac{Q_{\mathrm{T}, k}(t)}{T_{\mathrm{e} 0}} .
\end{gathered}
$$

If the heating source is switched off at $t=0$, the relaxation of medium-sized depletions for $\tau_{\mathrm{r}}>t>\tau_{\mathrm{T}}$ is determined according to (6.7) by ambipolar ion diffusion with coefficient $\left(1+T_{\mathrm{e} 0} / T_{\mathrm{i}}\right) D_{\perp}^{(\mathrm{i})}$ across the magnetic field and $\left(1+T_{\mathrm{e} 0} / T_{\mathrm{i}}\right) D_{\|}^{(\mathrm{i})}$ along the magnetic field.

To investigate the relaxation of small-scale irregularities $L_{\|}^{(N)} \ll L_{\perp}^{(\text {cr })}$ we substitute the relation $(6.5)$ into $(6.1),(6.3)$. The equations obtained can be presented in the form

$$
\begin{gathered}
\hat{L}_{1} \Delta n_{k}=-\left(2.5 k_{x}^{2} D_{\perp}^{(\mathrm{e})}+3.4 k_{z}^{2} D_{\|}^{(\mathrm{e})}\right) \Delta \tau_{k} \\
\hat{L}_{2} \Delta \tau_{k}+\left(1+\frac{T_{\mathrm{i}}}{T_{\mathrm{e} 0}}\right)\left(1.5 k_{x}^{2} D_{\perp}^{(\mathrm{e})}+1.4 k_{z}^{2} D_{\|}^{(\mathrm{e})}\right) \Delta n_{k}=\frac{Q_{\mathrm{T}, k}}{T_{\mathrm{e} 0}}
\end{gathered}
$$


where

$$
\begin{aligned}
& \hat{L}_{1}=\frac{\partial}{\partial t}+\left(1+\frac{T_{\mathrm{i}}}{T_{\mathrm{e} 0}}\right)\left(k_{x}^{2} D_{\perp}^{(\mathrm{e})}+2 k_{z}^{2} D_{\|}^{(\mathrm{e})}\right), \\
& \hat{L}_{2}=\frac{\partial}{\partial t}+\left(6.16 k_{x}^{2} D_{\perp}^{(\mathrm{e})}+7.54 k_{z}^{2} D_{\|}^{(\mathrm{e})}\right)+\frac{1}{\tau_{\mathrm{T}}} .
\end{aligned}
$$

The system $(6.9),(6.10)$ reduces to one equation describing the relaxation of a small-scale plasma perturbation

$$
\hat{L}_{2} \hat{L}_{1} \Delta n_{k}-\left(1+\frac{T_{\mathrm{i}}}{T_{\mathrm{e} 0}}\right)\left(2.5 k_{x}^{2} D_{\perp}^{(\mathrm{e})}+3.4 k_{z}^{2} D_{\|}^{(\mathrm{e})}\right)\left(1.5 k_{x}^{2} D_{\perp}^{(\mathrm{e})}+1.4 k_{z}^{2} D_{\|}^{(\mathrm{e})}\right) \Delta n_{k}=0 .
$$

If we seek the solution of the form $\Delta n_{k} \propto \exp \left[-\gamma\left(k_{x}, k_{z}\right) t\right]$, an equation of the second order with respect to $\gamma$ is obtained. This equation has two different roots $\gamma_{1,2}$ that describe relaxation with two time scales. To find the relaxation law in the usual space we need to apply the inverse Fourier transform. As the roots are rather complicated functions $\gamma_{1,2}=\gamma_{1,2}\left(k_{x}, k_{z}\right)$ the decay of a small-scale cavity does not obey the exponential law in the general case. It is important to mention that the estimate for the critical length $L_{\perp}^{\text {(er) }}$ and the peculiarities of the relaxation of small-scale and medium-sized irregularities are in qualitative agreement with the experimental data (Frolov et al. 1997). The detailed comparison with experiments is beyond the scope of the present paper and will be carried out elsewhere.

\section{Discussion and conclusions}

The distribution of plasma and the variation of the electron temperature in striations in stationary conditions were investigated analytically and numerically. It was found that in all cases the reduced plasma perturbation in striations $\Delta n$ is of the order of the variation of the electron temperature $|\Delta n| \sim \Delta \tau$. This means that it is impossible to expect a stationary state of striations with strongly enhanced electron temperature and very weak plasma depletion. The in-situ observations of Peria et al. (1999) and Kelley et al. (1995) at Arecibo indicate striations with plasma depletions on the order of $6 \%$ and electron temperature enhancements of $\sim 100 \mathrm{~K}$, which is likely to be of a similar order as the plasma depletion when the ambient electron temperature is taken into account. This is in good agreement with our theoretical predictions. At the same time this result does not prevent a significant increase of the electron temperature $T_{\mathrm{e} 0}$ within the heated volume compared to the undisturbed temperature. Such an increase is measured by incoherent scattering (Robinson et al. 1997; Leyser et al. 2000). A possible mechanism of anomalous heat transfer from striations to the whole heated volume will be discussed in a separate paper.

We have estimated the typical length of striations as $\sim(1-2) \times 10^{2} \mathrm{~km}$. This is much larger than estimated before (Rao and Thome 1974; Korovin et al. 1983; Jones et al. 1984; Kelley et al. 1995; Frolov et al. 1997). Such an elongation is connected to a strong localized heat outflow (mainly upward) along the magnetic field line.

Our conclusion is based on the assumption that the ionospheric turbulence in the heated volume does not influence the longitudinal diffusion of striations. This influence could be twofold. First, it might happen that striations are destroyed due 
to instabilities before they reach their stationary state. In this case, the real lifetime of striations is much smaller than the recombination lifetime $\tau_{\mathrm{r}}$. Second, due to the excitation of plasma turbulence in striations, the usual electron collision frequency $\nu_{\mathrm{ei}}$ should be substituted by some effective collision frequency $\nu_{\mathrm{e}}^{(\mathrm{eff})}$ which grows with the intensity of the excited waves and can exceed the frequency $\nu_{\mathrm{ei}}$ many times. Thus, the longitudinal diffusion and the length of striations are reduced significantly. To find confirmation of this effect, accurate measurements of the length of striations are desirable.

We have found numerically how the length of a striation depends on its transverse scale $L_{\perp}^{(N)}$. For small values of $L_{\perp}^{(N)}$ its increase causes the elongation of striations. This result is in accordance with the experimental data (Frolov et al. 1997). For the scales $L_{\perp}^{(N)}>5-6 \mathrm{~m}$, the length of a striation does not depend on its transverse scale. At the same time the elongation depends on the vertical scale of plasma inhomogeneity $L_{z}$ and the increase of the bulk temperature of electrons within the heated volume. The higher the temperature $T_{\mathrm{e} 0}$, the stronger the elongation of striations.

We have discussed the formation of seed medium-sized irregularities in the vicinity of striations. We predict that depletions start to grow at the heights where striations are formed and later on become more and more elongated due to the thermal self-focusing. The predicted dynamics of the medium-sized depletions and their elongation are in qualitative agreement with the experimental data (Hedberg et al. 1983; Bakhmet'eva et al. 1989).

Finally, we have investigated the relaxation of small-scale $L_{\perp}^{(N)}<L_{\perp}^{(\text {cr) }}$ artificial plasma irregularities (striations) and we have confirmed theoretically the appearance of two time scales that determine the decay of small-scale irregularities that is often seen in experiments (Frolov et al., 1997). A more detailed investigation of the relaxation of small-scale irregularities and comparison with observations will be the subject of a future paper.

\section{Acknowledgement}

AS is supported by a research studentship from the UK's Particle Physics and Astronomy Research Council.

\section{References}

Bakhmet'eva, N. V., Goncharov, N. P., Ignat'ev, Yu. A., Korotina, G. S., Tolmacheva, A. V. and Shavin, P. B. 1989 The spatial-temporal characteristics of inverse scattering signals from an artificial disturbed region. Geomagnet. Aeronomy 29, 701-705.

Blaunstein, N. 1996 Changes of the electron concentration profile during local heating of the ionospheric plasma. J. Atmos. Terr. Phys. 58, 1345-1354.

Blaunstein, N. 1997 Evolution of a stratified plasma structure induced by local heating of the ionosphere. J. Atmos. Solar-Terr. Phys. 59, 351-361.

Braginskii, S. I. 1965 Transport processes in a plasma. Reviews of Plasma Physics, Vol. 1. New York: Consultants Bureau, pp. 205-311.

Erukhimov, L. M., Metelev, S. A., Myasnikov, E. N., Mityakov, N. A. and Frolov, V. L. 1987 Artificial ionospheric turbulence. Radiophys. Quantum Electron. 31, 156-171.

Frolov, V. L., Erukhimov, L. M., Metelev, S. A. and Sergeev, E. N. 1997 Temporal behaviour of artificial small-scale ionospheric irregularities: review of experimental results. J. Atmos. Solar-Terr. Phys. 59, 2317-2333. 
Gondarenko, N. A., Guzdar, P. N., Milikh, G. M., Sharma, A. S., Papadopoulos, K. and Ossakow, S. L. 1999 Spatio-temporal development of the filaments due to the thermal self-focusing instability near the critical surface in ionospheric plasmas. Radiophys. Quantum Electron. 42, 589-600.

Gurevich, A. V. 1978 Nonlinear Phenomena in the Ionosphere. New York: Springer.

Gurevich, A. V., Lukyanov, A. V. and Zybin, K. P. 1995a Stationary state of isolated striations developed during ionospheric modification. Phys. Lett. A 206, 247-259.

Gurevich, A. V., Zybin, K. P. and Lukyanov, A. V. 1995b Stationary striations developed in the ionospheric modification. Phys. Rev. Lett. 75, 2622-2625.

Guzdar, P. N., Chaturvedi, P. K., Papadopoulos, K. and Ossakow, S. L. 1998 The thermal selffocusing instability near the critical surface in the high-latitude ionosphere. J. Geophys. Res. 103, 2231-2237.

Hansen, J. D., Morales, G. J., Duncan, L. M., Maggs, J. E. and Dimonte, G. 1990 Large-scale ionospheric modifications produced by nonlinear refraction of an HF wave. Phys. Rev. Lett. 65, 3285-3288.

Hedberg, A., Derblom, H., Thidé, B., Kopka, H. and Stubbe, P. 1983 Observations of HF backscatter associated with the Heating experiment at Tromsø. Radio Sci. 18, 840-850.

Jones, T. B., Robinson, T. R., Stubbe, P. and Kopka, H. 1984 Frequency dependence of anomalous absorption caused by high power radio waves. J. Atmos. Terr. Phys. 46, 147153.

Kadomtsev, B. B. and Pogutse, O. P. 1970 Turbulence in toroidal systems. Reviews of Plasma Physics, Vol. 5. New York: Consultants Bureau, pp. 249-400.

Kelley, M. C., Arce, T. L., Salowey, J., Sulzer, M., Armstrong, W. T., Carter, M. and Duncan, L. 1995 Density depletions at the $10 \mathrm{~m}$ scale induced by the Arecibo heater. J. Geophys. Res. 100, A9, 17367-17376.

Korovin, A. V., Nasyrov, A. M. and Yagnov, N. N. 1983 Space and frequency correlation of a field scattered by small-scale artificial irregularities of $\mathrm{F}$ ionosphere region. Radiophys. Quantum Electron. 26, 120-124.

Leyser, T. B., Gustavsson, B., Brändström, B. U. E., Steen, Å., Honary, F., Rietveld, M. T., Aso, T. and Ejiri, M. 2000 Simultaneous measurements of high-frequency pumpenhanced airglow and ionospheric temperature at auroral latitudes. Adv. Polar Upper Atmos. Res. 14, 1-11.

Minkoff, J., Kugelman, P. and Weissman, I. 1974 Radio frequency scattering from a heated ionospheric volume, VHF/UHF field-aligned and plasma-line backscatter measurements. Radio Sci. 9, 941-956.

Peria, W. J., Kelley, M. C. and Franz, T. 1999 Double-probe measurements in field-aligned irregularities produced by intense electromagnetic radiation. J. Geophys. Res. 104, A4, $6797-6804$.

Rao, P. B. and Thome, G. D. 1974 A model for RF-scattering from field-aligned heaterinduced irregularities. Radio Sci. 9, 987-996.

Robinson, T. R., Honary, F., Stocker, A. J., Jones, T. B. and Stubbe, P. 1996 First EISCAT observations of the modification of F-region electron temperatures during RF heating at harmonics of the electron gyro frequency. J. Atmos. Terr. Phys. 58, 385-395.

Schunk, R. W. and Nagy, A. F. 1978 Electron temperatures in the F region of the ionosphere: theory and observations. Rev. Geophys. Space Phys. 16(3), 355-399. 